\title{
A setup for ultrafast time-resolved $x$-ray absorption spectroscopy
}

\author{
Melanie Saes, ${ }^{\text {a) }}$ Frank van Mourik, Wojciech Gawelda, Maik Kaiser, Majed Chergui, \\ and Christian Bressler ${ }^{\mathrm{b})}$ \\ Université de Lausanne, IPMC-BSP, CH-1015 Lausanne-Dorigny, Switzerland \\ Daniel Grolimund \\ Nuclear Energy and Safety Research Department, Paul Scherrer Institut, CH-5232 Villigen-PSI, Switzerland \\ Rafael Abela \\ Swiss Light Source, CH-5232 Villigen-PSI, Switzerland \\ Thornton E. Glover, Philip A. Heimann, and Robert W. Schoenlein \\ Material Science Division, Advanced Light Source, Lawrence Berkeley National Laboratories, \\ 1 Cyclotron Road, Berkeley, California 94720 \\ Steven L. Johnson, ${ }^{\mathrm{c})}$ Aaron M. Lindenberg, and Roger W. Falcone \\ Department of Physics, 366 LeConte Hall, University of California, Berkeley, California 94720
}

(Received 17 July 2003; accepted 14 October 2003)

\begin{abstract}
We present a setup which allows the measurement of time-resolved x-ray absorption spectra with picosecond temporal resolution on liquid samples at the Advanced Light Source at Lawrence Berkeley National Laboratories. The temporal resolution is limited by the pulse width of the synchrotron source. We characterize the different sources of noise that limit the experiment and present a single-pulse detection scheme. (C) 2004 American Institute of Physics.
\end{abstract}

[DOI: $10.1063 / 1.1633003$ ]

\section{INTRODUCTION}

Ultrafast (femtosecond to picosecond) spectroscopy allows a better understanding of the possible pathways/ mechanisms of chemical reactions. ${ }^{1}$ In a pump-probe experiment, which is a typical scheme for ultrafast spectroscopy, the pump pulse triggers a chemical reaction and the probe pulse takes a snapshot of the ongoing reaction at a fixed time delay. By changing the time delay between pump and probe pulse, one can observe the development of the photoinduced reaction. A complete description of chemical dynamics ideally requires, besides ultrafast temporal resolution, a spatial resolution on the atomic scale. Furthermore, since many elementary reactions in chemistry and biology take place in a solvent environment, structural techniques, which can be implemented on solvated samples, are very desirable.

One method, which provides temporal resolution in the picosecond/femtosecond domain with atomic scale spatial resolution, is ultrafast time-resolved $\mathrm{x}$-ray absorption spectroscopy (XAS). In this type of pump-probe experiment, the probe pulse consists of an X-ray pulse, while an ultrashort laser pulse triggers the reaction. The $\mathrm{x}$-ray probe yields information on the oxidation state of an atom of interest and on the unoccupied valence states via $\mathrm{x}$-ray absorption near-edge structure spectroscopy and information on the local molecular structure around an atom of interest via extended $\mathrm{x}$-ray absorption fine structure (EXAFS). ${ }^{2}$

\footnotetext{
a) Also at: Swiss Light Source, CH-5232 Villigen-PSI, Switzerland.

${ }^{b)}$ Author to whom correspondence should be addressed; electronic mail: christian.bressler@ipmc.unil.ch

${ }^{c)}$ Current address: Swiss Light Source, CH-5232 Villigen-PSI, Switzerland.
}

Only a few fast and ultrafast time-resolved XAS measurements have been carried out so far. ${ }^{3-7}$ The $\mathrm{x}$-ray sources used in these studies were either laser driven plasma sources ${ }^{3}$ or synchrotrons. ${ }^{4-7}$ These experiments generally suffer from the small signals typical of laser-induced changes to $\mathrm{x}$-ray absorption spectra. ${ }^{8}$ Moreover, the photon flux of the x-ray source is a major limiting factor for the signal-to-noise ratio of the experiment. In the following, we limit our considerations to synchrotron radiation, which is currently the brightest and most stable and reliable source of tuneable hard $\mathrm{x}$ rays.

We report on a setup realized at beamline 5.3.1 at the Advanced Light Source (ALS) at Lawrence Berkeley National Laboratories. The focus will be on the single-pulse detection scheme that we use in our x-ray transmission measurements, which makes it possible to isolate and characterize the different noise sources that potentially limit the experiment. The data obtained allow us to evaluate the feasibility of future experiments.

\section{INSTRUMENTS AND METHOD}

Figure 1 displays the main elements required for a picosecond (ps) time-resolved $\mathrm{x}$-ray absorption experiment at a synchrotron. The different components consisting of x-ray source, beamline, laser system, and data acquisition will be described in the following.

\section{A. X-ray source}

Synchrotrons sources provide typically $50-100 \mathrm{ps}$ long pulses of polychromatic radiation. The repetition rate of the pulses depends on the filling pattern of the storage ring and its revolution frequency. At the ALS storage ring, which has a revolution frequency of $1.52 \mathrm{MHz}$, we make use of the 


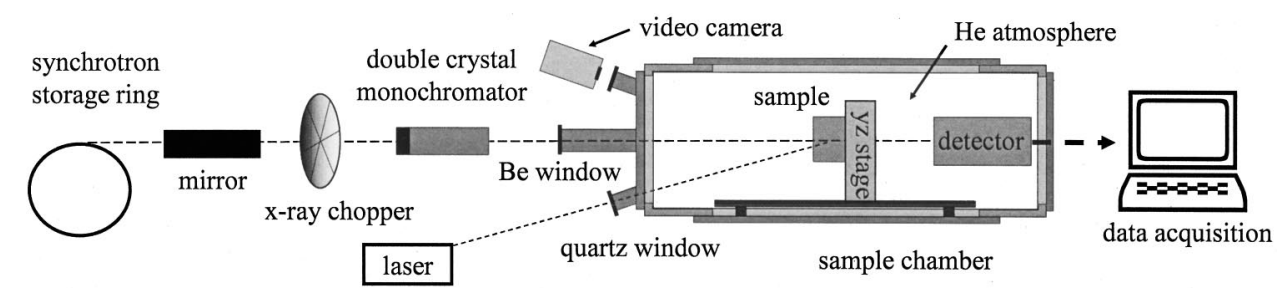

FIG. 1. Schematic setup for a laser-pump x-ray-probe experiment. X rays from a synchrotron are guided through a beamline into the sample chamber where they overlap with synchronized laser pulses at the sample position. The laser pulses excite the sample, while the $\mathrm{x}$ xrays probe pulses the photoinduced changes. A detection scheme in transmission mode records the x-ray pulse intensities.

"camshaft mode" operation, shown in Fig. 2. In this mode, the ring is filled with 276 electron bunches separated by 2 ns, each contributing $1.4 \mathrm{~mA}$ to the overall ring current. One isolated bunch, which contributes $10 \mathrm{~mA}$, is placed within the 100 ns empty section of the ring. We will refer to the 276 closely packed bunches as "multibunch" and the isolated bunch as the "camshaft pulse." A modestly fast x-ray detector (e.g., large area avalanche photodiode) can single out the radiation from the camshaft pulse (Fig. 3), which is therefore a suitable probe pulse for the time-resolved experiment. Note that the time-resolved experiment uses only a fraction of the available flux. The multibunch is completely ignored and, in addition, the repetition rate of the experiment depends on the usually lower repetition rate of the laser system. We use an amplified femtosecond laser system which delivers intense and ultrashort pump pulses at a repetition rate of $1 \mathrm{kHz}$. Thus, the useful flux of the synchrotron is reduced by a factor of $10^{5}$, which makes it, compared to the laser pump pulse ( $10^{15}$ photons), a weak probe pulse ( $10^{4}$ photons $)$.

\section{B. Advanced Light Source beamline 5.3.1}

Beamline 5.3.1 is a bend magnet beamline designed for various time-resolved experiments. The radiation is collected under a $5 \mathrm{mrad}$ grazing incidence angle by a toroidal platinum-coated mirror, which provides a 1:1 double focus $24 \mathrm{~m}$ from the source. The focus images the electron-beam source, approximately $50 \mu \mathrm{m}$ (vertical) $\times 250 \mu \mathrm{m}$ (horizon-

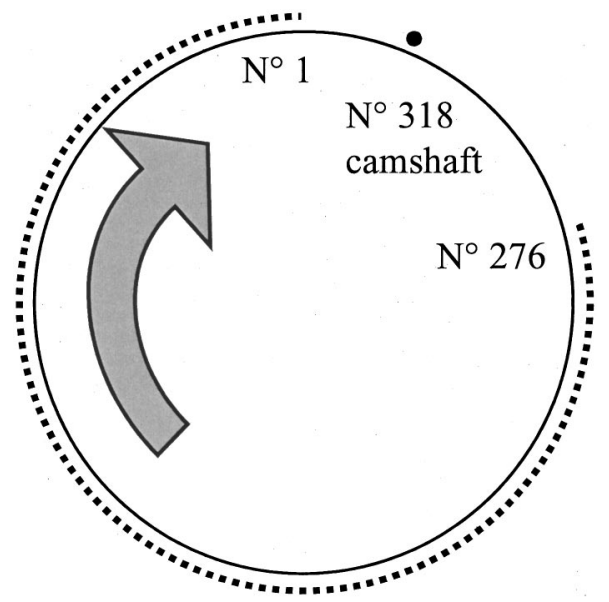

FIG. 2. Electron filling pattern used in our experiments at the ALS. In total, there are 328 possible electron buckets. In the camshaft mode at the ALS buckets 1 to 276 are filled and a single pulse, the camshaft pulse, is placed in the gap between the empty buckets 277 and 328 (typically bucket No. 318). tal). A Ge(111) double monochromator with fixed output beam height (which is located $2 \mathrm{~m}$ upstream from the focus) selects monochromatic $x$ rays from $2.2 \mathrm{keV}$ to $12 \mathrm{keV}$ with an energy resolution of $\Delta E / E=3 \times 10^{-4}$ to $1 \times 10^{-3}$ bandwidth, respectively (with $E$ being photon energy). A mechanical chopper placed before the monochromator cuts out an average of $95 \%$ of the $\mathrm{x}$-ray flux to reduce the overall heatload on the first monochromator crystal. Details of the beamline setup are given in Ref. 9.

\section{Sample chamber}

The $\mathrm{x}$ rays enter the sample chamber (Fig. 1) through a $50 \mu \mathrm{m}$ thick beryllium window. The window separates the ultrahigh vacuum of the beamline from the chamber, which is purged with helium at atmospheric pressure. The laser enters through a quartz window on the beamline side of the chamber. Another window allows the monitoring of the sample with a video camera. The sample (see below) is mounted on a motorized $y z$ translation stage for adjusting its position within the $\mathrm{x}$-ray beam. The $\mathrm{x}$-ray detector is placed about $20 \mathrm{~cm}$ behind the sample for transmission signal measurements.

\section{Sample}

Our samples are liquid solutions. A sapphire nozzle creates a thin, flat jet at the point where $\mathrm{x}$ ray and laser beams overlap, as indicated in Fig. 4. We typically choose a nozzle so as to obtain a thickness of the jet of 100-500 $\mu \mathrm{m}$, depending on the experiment. ${ }^{8}$ The liquid jet has a velocity of $\sim 5 \mathrm{~m} / \mathrm{s}$, which is fast enough to ensure the renewal of the sample at the repetition rate of the measurement. The liquid from the jet is caught and recycled by passing through a reservoir, also kept under a helium atmosphere.

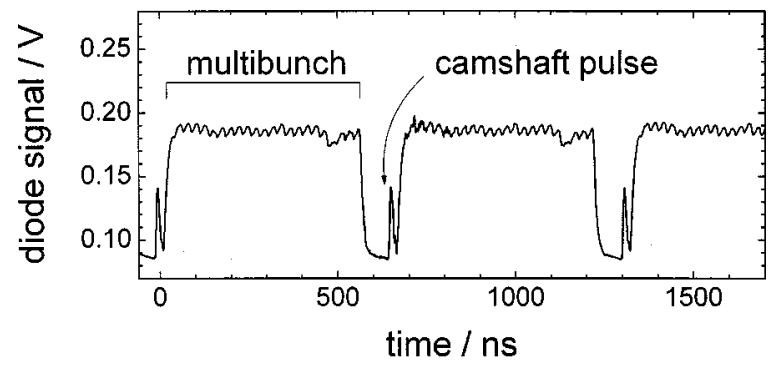

FIG. 3. Oscilloscope trace of the x-ray train recorded by a large area avalanche photodiode. Due to its slower response its signal on the multibunch train accumulates for $\sim$ ten bunches, which in return lets the much more intense single-camshaft pulse appear smaller in the figure. 


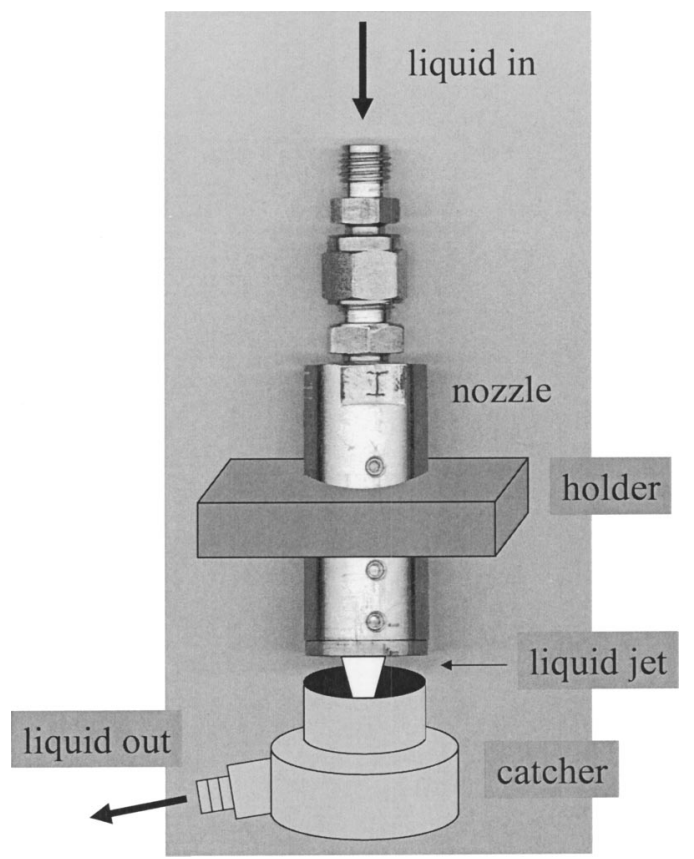

FIG. 4. Sapphire nozzle creating a thin liquid jet of the sample. The displacement of the sample is possible in the plane perpendicular to the incident $\mathrm{x}$ rays.

\section{E. Laser source}

High intensity laser pulses are needed to excite a significant fraction of the sample. ${ }^{8,10}$ The laser pulse length should be similar to the $\mathrm{x}$-ray probe length or shorter in order to keep the temporal resolution as high as possible. We use a Ti-sapphire-based amplified femtosecond laser system (Spectra-Physics) producing $150 \mathrm{fs}$ pulses at $800 \mathrm{~nm}$ with a pulse energy of $\sim 2 \mathrm{~mJ}$ at a repetition rate of $1 \mathrm{kHz}$. By second-harmonic generation in a BBO crystal $(12 \times 12 \times 0.5$ $\mathrm{mm}$, type $1, \theta=29^{\circ}, \varphi=90^{\circ}$ ), we typically obtain $500 \mu \mathrm{J} /$ pulse at $400 \mathrm{~nm}$.

A pump-probe experiment relies on the reproducibility of the time delay between pump and probe pulses. Therefore, the laser has to be accurately synchronized to the $\mathrm{x}$-ray source. The laser oscillator is synchronized to the $500 \mathrm{MHz}$ radio frequency (rf) of the storage ring by adjusting the oscillator cavity length (and thus its frequency) proportionally to the phase error between the rf and the sixth-harmonic of the oscillator output. ${ }^{11,12}$ The laser amplifier is synchronized to the revolution frequency of the storage ring and the opening window of the $x$-ray chopper. Thus, a seed pulse from the oscillator will be selected and amplified every millisecond, which coincides with a particular $\mathrm{x}$-ray pulse (the camshaft pulse) on the sample.

The delay between x-ray and laser pulses can be adjusted in coarse steps of $12 \mathrm{~ns}$ by selecting different seed pulses from the laser oscillator. For finer tuning, a computercontrolled phase shifter (I.F. Engineering, model QPMX499) is used to adjust the phase of the reference rf signal, which synchronizes the laser oscillator. ${ }^{13}$ The jitter between laser and $\mathrm{x}$-ray pulses was estimated to be less than $10 \mathrm{ps}{ }^{14}$ Typical values reported for the jitter between a laser oscillator and a synchrotron or free electron laser source are on the order of 1 ps or less. ${ }^{11,12}$
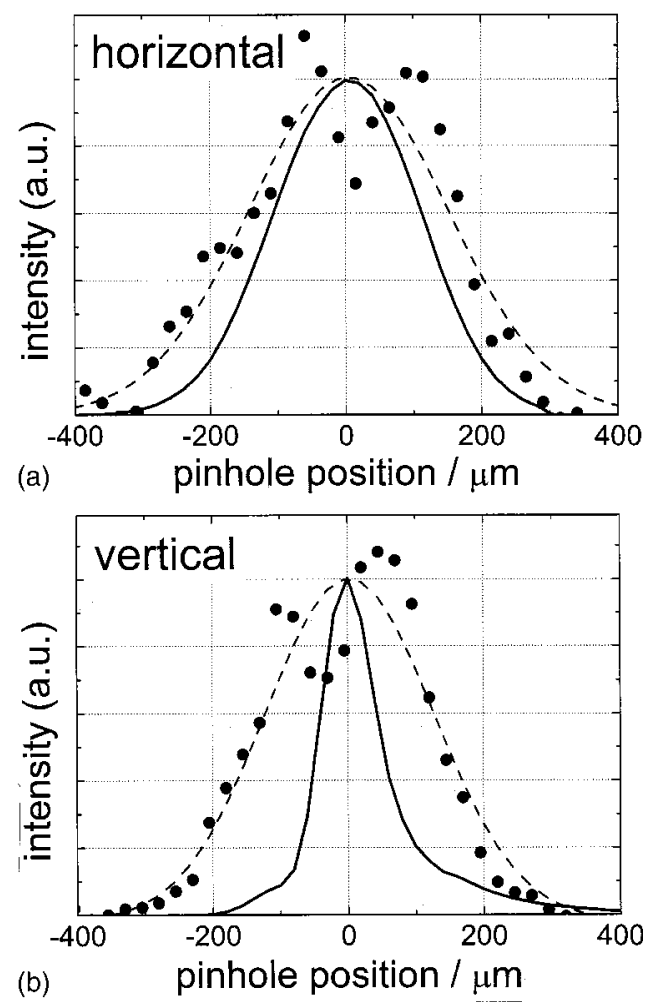

FIG. 5. X-ray (solid line) and laser beam profiles (dotted and dashed curves as fit) scanned through a $50 \mu \mathrm{m}$ diameter pinhole.

\section{F. Spatial overlap}

The spatial overlap between $\mathrm{x}$-ray and laser pulses is obtained by replacing the sample with a $50 \mu \mathrm{m}$ diameter pinhole on a $25 \mu \mathrm{m}$ thick tungsten substrate that is placed at the exact position of the jet. A two-dimensional translation stage (Fig. 1) moves the pinhole across the x-ray beam, while an $\mathrm{x}$-ray transmission measurement maps out the beam profile. The pinhole is centered on the $\mathrm{x}$-ray beam and the laser is then guided through the pinhole. The angle between laser and $\mathrm{x}$-ray beam is about $15^{\circ}$. Figure 5 shows the vertical and horizontal beam profiles of the $\mathrm{x}$-ray and the laser beams at the sample position. The $\mathrm{x}$-ray beam size is vertically $100 \mu \mathrm{m}$ and horizontally $260 \mu \mathrm{m}$. The laser spot has a circular shape and was adjusted in size to the horizontal diameter of the x-ray spot.

\section{G. Temporal overlap}

For setting the temporal overlap, we place a fast windowless photodiode (rise time $\sim 0.7 \mathrm{~ns}$ ) at the sample position and record both the laser and the $\mathrm{x}$-ray pulses. Using a 1 $\mathrm{GHz}$ oscilloscope, we adjust the timing to within a few hundred picoseconds. During the experiment, the relative timing of camshaft and laser pulses is monitored constantly by simultaneously measuring the laser pulses and visible synchrotron pulses from another beamline at the same bend magnet.

For defining the temporal overlap more precisely, a pump-probe signal of a sample is used. A suitable sample to achieve this is $\left[\mathrm{Ru}(\mathrm{bpy})_{3}\right]^{2+}$ in water. In this case, a signal is generated by a $400 \mathrm{~nm}$ pulse in a $0.1 \mathrm{~mm}$ thick free flow- 


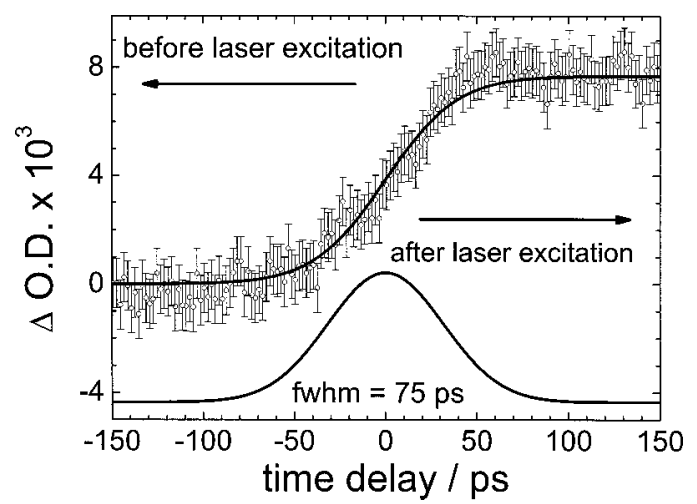

FIG. 6. Pump-probe signal of aqueous $\left[\mathrm{Ru}^{\mathrm{II}}(\mathrm{bpy})_{3}\right]^{2+}$ together with a fit as a function of pump-probe time delay. The onset of the photoinduced signal $(<300 \mathrm{fs})$ is comparable to a step function; thus, the rise we measure is a convolution of the pump-probe effect with the width of the synchrotron pulse. The lower curve is the derivative of the fit curve (which yields a Gaussian) whose width corresponds to the cross-correlation signal, which is governed by the indicated $\mathrm{x}$-ray pulse width.

ing water jet of $80 \mathrm{mM}\left[\mathrm{Ru}(\mathrm{bpy})_{3}\right]^{2+}$. Upon excitation, the central ion of the compound changes its oxidation state according to

$$
\left[\mathrm{Ru}^{\mathrm{II}}(\mathrm{bpy})_{3}\right]^{2+}+400 \mathrm{~nm} \rightarrow\left[\mathrm{Ru}^{\mathrm{III}}(\mathrm{bpy})_{2}(\mathrm{bpy})^{-}\right]^{2+} .
$$

This product is created on a sub-ps time scale, and decays on a time scale of a few hundred ns. ${ }^{15}$ On a ps time scale, the onset of the photoinduced reaction is comparable to a step function. Setting the monochromator to $2840 \mathrm{eV}$ and monitoring the valence shift of the $\mathrm{Ru} L_{3}$ edge as a function of pump-probe delay, ${ }^{7}$ we obtain a pump-probe signal, which shows this step function convoluted with the x-ray probe pulse length. The scan, as shown in Fig. 6, yields a cross correlation time of $75 \pm 10 \mathrm{ps}$, which includes the actual $\mathrm{x}$-ray pulse width and the synchronization jitter. The accuracy of fitting the slope of the signal onset in Fig. 6 defines an upper limit for the synchronization jitter. The error bars represent the sum of all noise sources including the synchronization jitter in the region of the signal onset. Since the error bars on the plateaus before and after time zero have the same magnitude as the error bars in the signal onset, we conclude that the noise introduced by the jitter is not dominant. Therefore, with the given error bars, we can make an upper estimate for the synchronization jitter, which is \pm 10 ps. Higher accumulation times should narrow down the error bars and allow a better estimate.

\section{H. Detection/data acquisition}

The detector is a windowless APD (Advanced Photonix, model 394-70-72-500). The Avalanche photodiode (APD) is mounted in a sealed metal box with a $15 \mu \mathrm{m}$ thick beryllium window over the active area, which protects the diode from the intense scatter of the laser pulse. In addition, a $10 \mu \mathrm{m}$ foil of mylar protects the beryllium window from the humidity created by the aqueous sample upon laser excitation. The rise time of the APD is $12 \mathrm{~ns}$, which is sufficiently fast to measure the isolated camshaft pulse (Fig. 3).

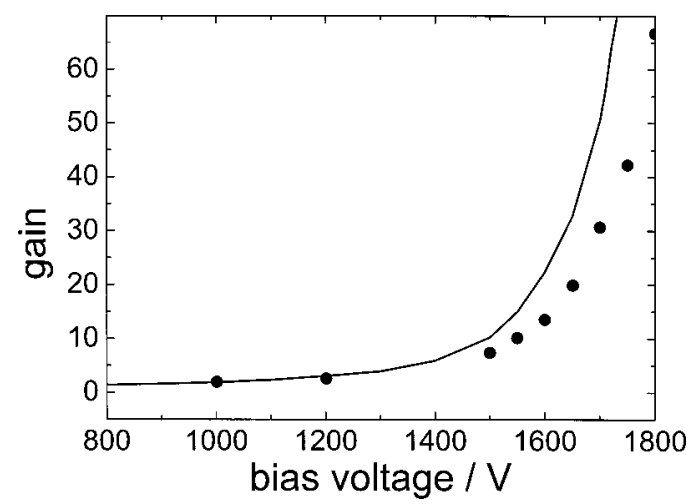

FIG. 7. APD gain curve for visible (line) and x-ray (dots) pulses. The lower gain for X-ray pulses is attributed to space-charge effects and local heating in the avalanche region of the detector.

In order to estimate the gain of the detector, we measured the output of the APD as a function of bias voltage under two different conditions:

(1) $80 \mathrm{fs}$ pulses of $1.5 \mathrm{eV}$ visible photons at $1 \mathrm{kHz}$ pulse repetition rate, and

(2) $3000 \mathrm{eV}$ x-ray photons from the camshaft pulse.

The curves are plotted in Fig. 7. They were normalized to the signal at low bias, corresponding to unit gain. The signals obtained with laser light are in excellent agreement with the curve provided by the manufacturer, over the whole range of bias settings.

The deviation at higher bias voltages between visible light and X-ray measurements has been reported before, ${ }^{16,17}$ and was attributed to space-charge effects decreasing the electrical field in the avalanche region and local heating by the high-energy x-ray pulses. Whereas a visible photon produces only a single electron, an x-ray photon produces on the order of 1000 electrons in a very confined volume, which could locally cause a reduced gain. Note that due to the local nature of the effect, this need not give rise to an overall nonlinear response. As long as the X-ray photons are spread over a sufficiently large area of the detector, the electron pulses produced by different x-ray absorption events are independent of each other and only affect their own local gain. Therefore, the measured signal should still be proportional to the number of x-ray photons on the detector.

Indeed, the linear response of the APD (bias voltage $1750 \mathrm{~V}$ ) was verified by measuring the signal from the camshaft pulse as a function of the camshaft current (data provided by the ALS), and both were found to be proportional (see Fig. 8). According to our measurements, the linearity of the detector is given to at least 13000 photons per pulse detected within an estimated area of $150 \mu \mathrm{m} \times 600 \mu \mathrm{m}$. When carrying out this experiment at an undulator beamline, which has about 10-100 times larger pulse intensities, it may be necessary to move the detector further away from the $\mathrm{x}$-ray focus, so as to make use of a bigger spot size on the detector.

Figure 9 introduces the detection scheme used at beamline 5.3.1. In the experiment, the detector signal was amplified by a factor of 5 in a fast preamplifier (Stanford Research 


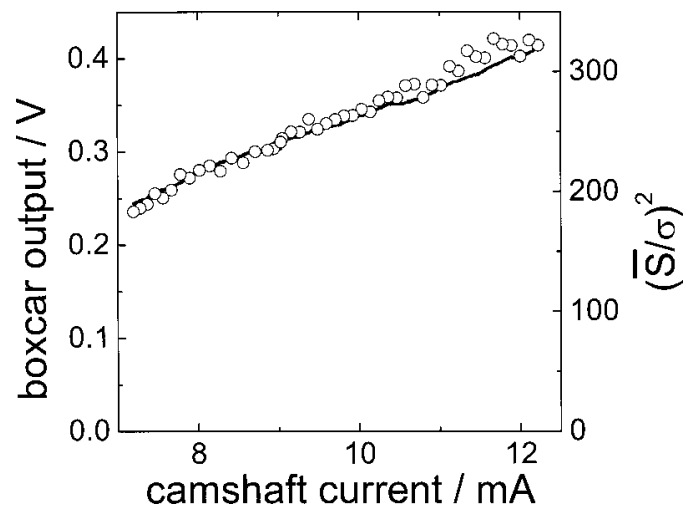

FIG. 8. The x-ray transmission signal (at $2840 \mathrm{eV}$ ) as given by the gated integrator output is plotted vs the camshaft current (line). The linearity between camshaft intensity and detector signal is apparent. The squared signalto-noise ratio $(\bar{S} / \sigma)^{2}$ (dots) follows the same trend and indicates that the detection scheme is mainly limited by the shot noise of the x-ray source.

Systems, SR445). The amplified signal from the camshaft pulse was picked out using a gated integrator (Stanford Research Systems, SR250), and digitized using an analog-todigital converter (National Instruments, PCI-6035E). In our experiment, the difference spectra are more important than the actual x-ray absorption spectra; therefore, we used the most simple detection scheme, using a single-x-ray detector without a reference detector before the sample. To get a difference signal, we detect the camshaft pulse at twice the repetition rate of the laser, so the detector alternately measures the excited and unexcited samples once. Because both signals are subtracted to give the difference signal, slow drifts in the detector or electronics are effectively eliminated. Simultaneously, with the APD signal, a reference signal from the laser trigger is digitized. This allows sorting out the laserexcited and unexcited transmission signals. An interesting side effect of this detection technique is its insensitivity to slow (minutes to hours) sample degradation. Because reference spectra are taken within short intervals of $500 \mu \mathrm{s}$, the difference signal induced by the photoexcitation will be unaffected, provided that the photodissociation products exhibit no additional photoinduced $\mathrm{x}$-ray absorption changes.

There is an advantage in further increasing the repetition rate of the measurement to $4 \mathrm{kHz}$. There is no baseline drift on the difference spectra, but there is one for the individual excited and unexcited spectra. So, when we want to compare the height of the pump-probe effect to the absolute transmission signal to estimate the overall effect, it would be profitable to actually measure the magnitude of the baseline drift on a shot-to-shot basis. When measuring at four times the laser frequency $(4 \mathrm{kHz})$, the $2 \mathrm{kHz}$ x-ray chopper cuts out the $\mathrm{x}$-ray flux for every second data point allowing the measurement of the zero offset. Thus, we measure the pump-probe signal with high precision as

$$
\log \frac{I_{\text {unexcited }}-I_{\text {unexcited }}^{0}}{I_{\text {excited }}-I_{\text {excited }}^{0}},
$$

where $I_{\text {excited }}$ and $I_{\text {unexcited }}$ are the transmission signals through the laser-illuminated and dark samples, respectively; $I_{\text {excited }}^{0}$ and $I_{\text {unexcited }}^{0}$ are the corresponding zero offsets (without $\mathrm{x}$ rays). The transformation of the static transmission spectra into absorption spectra via

$$
\log \frac{I_{0}}{I_{\text {unexcited }}}
$$

is carried out more roughly by scanning the x-ray energy once with $\left(I_{\text {unexcited }}\right)$ and once without $\left(I_{0}\right)$ the sample in the beam.

During an energy or a time-delay scan, the data acquisition process is set to a halt until the new experimental conditions have settled, i.e., while the monochromator is moving or the laser timing is adjusted, no data are recorded.

In order to discriminate between electronic noise in our detection scheme and true fluctuations in the x-ray flux, we measured the pulse-height distribution of the APD output with and without $\mathrm{x}$ rays on the detector. The left-hand side curve in Fig. 10 shows the pulse-height distribution with the $\mathrm{x}$-ray shutter closed, which gives a measure for the total electronic noise in our detection system. In this example, the distribution measured with a typical flux of $\mathrm{x}$ rays on the detector is about 20 times higher. If we make the reasonable assumption that the electronic noise is independent of the photon statistics, then the electronic noise in the x-ray mea-

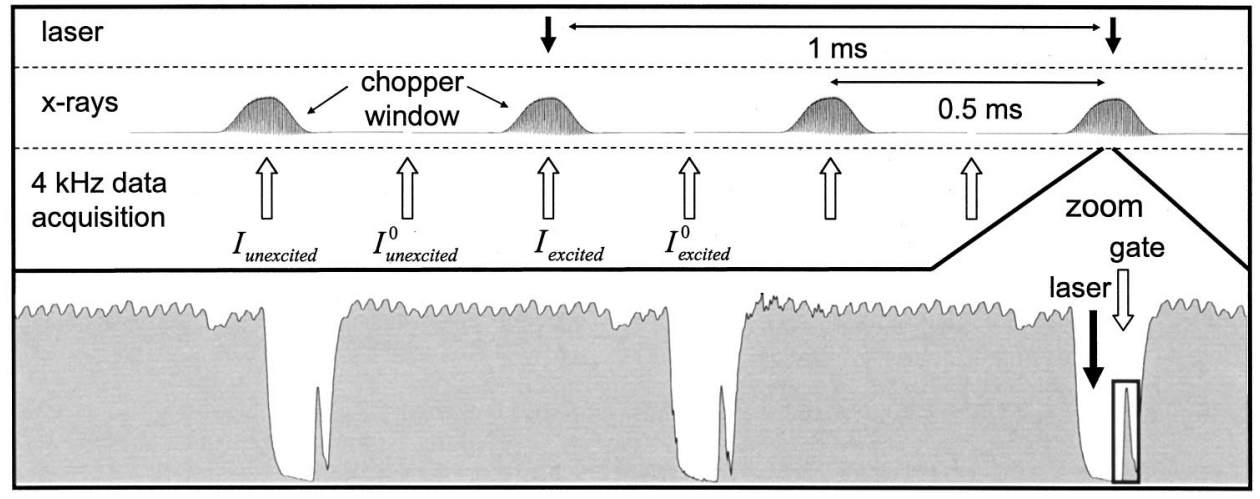

FIG. 9. $4 \mathrm{kHz}$-based setup for the data acquisition. The black arrows represent the $1 \mathrm{kHz}$ repetition rate laser pump pulse. A $2 \mathrm{kHz}$ chopper window (black, Gaussian features) allows $\mathrm{x}$ rays on the sample for every second detection gate. The white arrows represent the detection gate synchronized to the camshaft pulse. By reading out data within and without the chopper window, the absolute zero offset $\left(I_{\text {excited }}^{0}\right.$ and $\left.I_{\text {unexcited }}^{0}\right)$ can be measured. Every second chopper window coincides with a laser pulse $\left(I_{\text {excited }}\right)$, while the other serves as a reference $\left(I_{\text {unexcited }}\right)$. In this way, slow noise components on the data can be eliminated. In the lower part of the figure, we show a close-up view of the detection gate on the camshaft pulse with a preceding laser-pump pulse. 


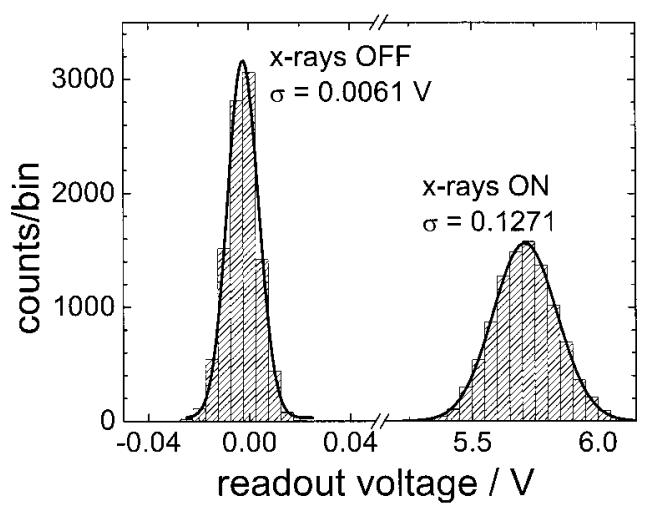

FIG. 10. Camshaft pulse-height distribution with $\mathrm{x}$ rays $\mathrm{ON}$ and OFF. The noise contribution from the setup ( $\mathrm{x}$-ray OFF) is negligible compared to the noise in the photon flux.

surement is negligible since the total noise is calculated from the square root of the sum of the squared standard deviations. In the case of the example in Fig. 10, reducing the electronic noise to zero would only reduce the overall noise from $0.1271 \mathrm{~V}$ to $0.1270 \mathrm{~V}$. Note that the pulse-height distribution is very well described by a Gaussian distribution.

By measuring the noise in the photon flux as a function of the flux (Fig. 8), we find a square law dependence of the noise, which is characteristic for shot noise. For other sources of noise, e.g., noise entering through variations in the throughput of the beamline optics (due to vibrations), we would expect a deviation from the square law dependence. Therefore, we conclude that no other significant noise sources play a role, and that, consequently, our noise measurement should be a precise measure for the absolute photon flux.

We calculate the photon flux via the measurement of the pulse-height distribution and application of the equation:

$$
\left(\frac{\bar{S}}{\sigma}\right)^{2}=\text { photons/pulse, }
$$

where $\bar{S}$ is the average signal of the pulse-height distribution and $\sigma$ is the corresponding standard deviation. With a camshaft current of $4.6 \mathrm{~mA}$ at an x-ray energy of $2850 \mathrm{eV}$, we measured a standard deviation of $0.0374 \mathrm{~V}$ on a signal of $2.0209 \mathrm{~V}$. For the shot-noise limited case, this corresponds to 2915 photons per pulse on the detector. Correction for the transmission of beryllium windows (total of $65 \mu \mathrm{m}, T$ $=74 \%)$, helium $(50 \mathrm{~cm}, T=98 \%)$, and mylar $(10 \mu \mathrm{m}, T$ $=81 \%$ ) and the normalization to $10 \mathrm{~mA}$ camshaft current yields $1.1 \times 10^{4}$ photons at the end of the beamline. This compares to $9 \times 10^{3}$ photons/pulse, which are the specifications of the beamline. ${ }^{18}$ Thus, we can conclude that the absolute photon flux can be measured with our setup and that the measurements are indeed shot-noise limited.

With this arrangement, we can record the x-ray absorption spectrum of the transient species of $\left[\mathrm{Ru}(\mathrm{bpy})_{3}\right]^{2+}$ in a pump-probe scheme (see Fig. 11 and Ref. 7). Figure 11(a) shows the static absorption spectrum around the ruthenium $L_{3}$ edge. Figure 11(b) presents the laser-induced absorption changes recorded at a fixed time delay of $60( \pm 10) \mathrm{ps}$. The detailed interpretation of the chemical processes, which are

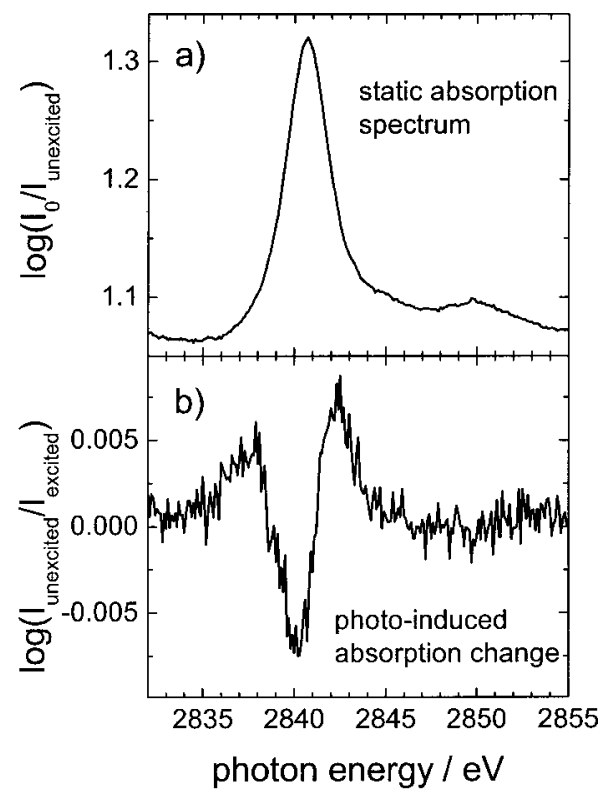

FIG. 11. (a) Static absorption spectrum of aqueous $\left[\mathrm{Ru}(\mathrm{bpy})_{3}\right]^{2+}$ showing the $\mathrm{Ru} L_{3}$ edge. (b) Laser-induced absorption changes recorded at a fixed delay of 60 ps (see Ref. 7 for details).

mirrored in the spectral changes, is given elsewhere., ${ }^{70}$ Here, we focus on the evaluation of the detection sensitivity of our setup. The noise level on the difference spectrum corresponds to a detection sensitivity of $1 \mathrm{mOD}$, which allows the detection of the transient changes in the ruthenium sample with a signal-to-noise ratio of 10 . The number of incident photons is $5 \times 10^{6}$ photons/data point, which corresponds to an integration over 2000 camshaft pulses per data point. The overall energy scan takes $15 \mathrm{~min}$. This is a reasonable integration time over which the experimental parameters (e.g., synchronization and laser power) can easily be stabilized. The excitation yield of the sample is on the order of $10 \%{ }^{7}$ By increasing the integration time, the sensitivity of the setup can be pushed down into the sub-mOD regime, which would allow the study of smaller pump-probe effects like measurements on dilute samples ${ }^{10}$ or the study of molecular structural changes via EXAFS, as proposed earlier. ${ }^{8}$

\section{DISCUSSION}

We have developed a setup at a synchrotron radiation source, which is capable of monitoring laser-induced transient $\mathrm{x}$-ray absorption with ps temporal resolution, and a sensitivity that allows the measurement of changes in $\mathrm{x}$-ray absorption on the order of $1 \mathrm{mOD}$. The statistical analysis reveals that the noise in the $\mathrm{x}$-ray probe signal is fully governed by the shot noise of the x-ray photon source.

The limiting factor of the temporal resolution of this setup is the duration of the synchrotron pulse. By exploiting the femtosecond-slicing technique, ${ }^{19}$ which is now in commission at the ALS, and under construction at the Swiss Light Source (SLS), we could descend to the hundreds of femtosecond time domain. This way, the temporal resolution of our laser pump-x-ray probe experiment would improve by three orders of magnitude. The available flux from the slicing technique, as employed by the SLS, has been esti- 
mated to deliver about $100-1000$ photons/pulse to the beamline. Therefore, we envisage the possibility of performing our experiments with this source with a large gain in temporal resolution, at the expense of a moderate reduction in the signal-to-noise ratio.

\section{ACKNOWLEDGMENTS}

The authors would like to thank Dr. Alexander Tarnovsky, Dr. Bernhard Lang, Dr. Jan Helbing, Dr. Philip Pattison, Dr. Marc Hertlein, Dr. Ali Belkacem, Dr. Inuk Kang, and Dr. Thomas Missalla for their help in this project. This work was financed by the FNRS via Contract Nos. 62066145.01 and 2000-067912-02/1, by the Swiss Light Source, by the Advanced Light Source via DOE Contract No. DEAC03-76SF00098, and by the University of Lausanne.

${ }^{1}$ Femtochemistry and Femtobiology: Ultrafast Dynamics in Molecular Science, edited by A. Douhal and J. Santamaria (World Scientific, Singapore, 2002).

${ }^{2} X$-ray Absorption Spectroscopy, edited by D. C. Koeningsberger and R. Prins (Wiley, New York, 1988).

${ }^{3}$ F. R. Raksi, K. R. Wilson, Z. Jiang, A. Ikhlef, C. Y. Cote, and J.-C. Kieffer, J. Chem. Phys. 104, 6066 (1996).

${ }^{4}$ L. X. Chen, W. J. H. Jäger, G. Jennings, D. J. Gosztola, A. Munkholm, and J. P. Hessler, Science 292, 262 (2001).

${ }^{5}$ L. X. Chen, G. B. Shaw, I. Novozhilova, T. Liu, G. Jennings, K. Attenkofer, G. J. Meyer, and P. Coppens, J. Am. Chem. Soc. 125, 7022 (2003).
${ }^{6}$ B. W. Adams, M. F. DeCamp, E. M. Dufresne, and D. A. Reis, Rev. Sci. Instrum. 73, 4150 (2002).

${ }^{7}$ M. Saes, C. Bressler, R. Abela, D. Grolimund, S. L. Johnson, P. A. Heimann, and M. Chergui, Phys. Rev. Lett. 90, 047403 (2003).

${ }^{8}$ C. Bressler, M. Saes, M. Chergui, D. Grolimund, R. Abela, and P. Pattison, J. Chem. Phys. 116, 2955 (2002).

${ }^{9}$ P. A. Heimann, A. M. Lindenberg, I. Kang, S. L. Johnson, T. Missalla, Z. Chang, R. W. Falcone, R. W. Schoenlein, T. E. Glover, and H. A. Padmore, Nucl. Instrum. Methods Phys. Res. A 467, 986 (2001).

${ }^{10}$ M. Saes, W. Gawelda, M. Kaiser, A. N. Tarnovsky, C. Bressler, M. Chergui, S. L. Johnson, D. Grolimund, and R. Abela, Synchrotron Radiat. News 16, 12 (2003).

${ }^{11}$ M. J. W. Rodwell, D. M. Bloom, and K. J. Weingarten, IEEE J. Quantum Electron. 25, 817 (1989).

${ }^{12}$ G. M. H. Knippels, M. J. van de Pol, H. P. M. Pellemans, P. C. M. Planken, and A. F. G. van der Meer, Opt. Lett. 23, 1754 (1998).

${ }^{13}$ S. L. Johnson, Ph.D. thesis, University of California, Berkeley, 2002.

${ }^{14}$ A. M. Lindenberg, Ph.D. thesis, University of California, Berkeley, 2001.

${ }^{15}$ N. H. Damrauer, G. Cerullo, A. Yeh, T. R. Boussie, C. V. Shank, and J. K. McCusker, Science 275, 54 (1997).

${ }^{16}$ M. Moszynski, M. Szawlowski, M. Kapusta, M. Balcerzyk, and D. Wolski, IEEE Trans. Nucl. Sci. 47, 1297 (2000).

${ }^{17}$ E. M. Gullikson, E. Gramsch, and M. Szawlowski, Appl. Opt. 34, 4662 (1995).

${ }^{18}$ T. E. Glover, P. A. Heimann, and R. W. Schoenlein, Advanced Light Source, Lawrence Berkeley National Laboratories, Berkeley, California Internal Report, "Beamline 5.3.1 Spectrally-Resolved Flux" (March 2002).

${ }^{19}$ R. W. Schoenlein, S. Chattopadhyay, H. H. W. Chong, T. E. Glover, P. A. Heimann, C. V. Shank, A. A. Zholents, and M. S. Zolotorev, Science 287, 2237 (2000). 